\title{
Exposé général sur la nappe alluviale de la Basse-Durance
}

\section{General notes on the alluvial water table in the lower Durance Valley}

PAR

A. DECELLE

DIRECTEUR A LA DIRECTION GÉNÉRALE D'ÉLECTRICITÉ DE FRANCE

\author{
R. MULLER-FEUGA \\ INGÉNIEUR A ÉLECTRICITÉ DE FRANCE \\ DIRECTION DE L'ÉQUIPEMENT
}

\begin{abstract}
L'établissement d'un projet hydroélectrique tel que celui de la dérivation de la basse Durance doit tenir compte de nombreux aspects dont certains sont liés an débeloppement agricole très poussé de cette partie de la vallée et à l'importance des débits prélevés pour les irrigations. Ce sont ces aspects qui ont conduit à étudier le régime actuel d'écoulement des eaux souterraines, le moyen susceptible d'estimer une conséquence éventuelle de la dérivation, lorsqu'elle sera créée, sur ce régime et les dispositions techniques propres à pallier, si besoin était, une telle conséquence. De semblables études, qui portent silr $90 \mathrm{~km}$ de vallée, nécessitent, pour être réalisées, un temps d'observation prolongé, un réseau de points de mesures suffisamment développé et de nombreux forages indispensables à la détermination des caractéristiques hydrodynamiques du milien aquifìre alluvial. Elles ont entrainé des recherches portant sur le régime non permanent des écoulements en milieu poreux ainsi que sur l'application de certaines methodes statistiques, et ont abouti, entre autres réalisations, $\dot{a}$ des essais d'alimentation artificielle de la nappe alluviale.
\end{abstract}

C'est depuis un siècle seulement que les travaux réalisés sur la Durance -- digues, ponts ont ébranlé la réputation de fléau que cette rivière au régime torrentiel avait acquise en Provence. De nos jours, elle est toutefois redontée encore par ses riverains : elle n'est en effet pratiquement pas régularisée. Ses excellentes caractéristiques de pente et de débit, qui en font une source d'énergie exceptionnelle ( 6 milliards de $\mathrm{kWh}$ ), sont par ailleurs à peine exploitées $(0,9$ milliards de $\mathrm{kWh})$.

La création du réservoir de Serre-Poncon et la dérivation de la Basse-Durance vers l'étang de

\begin{abstract}
When starting hydro-electric scheme like the Lower Durance diversion project various aspects have to be considered some of which are very closely bound up with the highlydeveloped agricultural activities in that part of the valley and with the large amounl of water taken off for irrigation purposes. Such considerations lead to the present ground water flow regimen being studied as a means of finding out what would be the likely effect of the diversion on the regimen and what arrangements would have to be made to off set such consequences if they arose. Studies of this kind which talie in a 90 kilometre length of the valley must necessarily include observations extending over a prolonged period. Also necessary is an adequate pattern of measurement stations and a large number of boreholes which are indispensable when determining the hydrodynamic characteristics of the waterbearing alluvial strata. The studies gave rise to research on non-permanent flow regimens in porous media as well as to the application of certain statistical methods and among other things resulted in tests on artificially feeding the alluvial ground water table.
\end{abstract}

Berre remédieront partiellement à cet état (3 milliards de $\mathrm{kWh}$ ).

Ce dernier aménagement doit se développer dans une région de plaines alluviales où l'agriculture est notoirement prédominante : il a fallu de ce fait prendre toutes les précautions désirables et montrer qu'il serait techniquement possible de pallier une éventuelle conséquence de la rérivation de la rivière sur la nappe alluviale dont la profondeur sous le sol varie entre 0 et $15 \mathrm{~m}$.

Les pages suivantes se proposent de décrire les études qui ont porté sur cette nappe alluviale ainsi que les essais auxquels elles ont abouti. 


\section{I. - DONNÊES GÉNÉRALES}

La Durance a un bassin versant de $14.127 \mathrm{~km}^{2}$ el draine ainsi plus du quart des Alpes francaises. Pour plusieurs raisons, on peut considérer que la Basse-Durance commence en aval du confluent du Verdon (figure 1) : en ce point situé à $90 \mathrm{~km}$ de son confluent avec le Rhône, la Durance a reçu son dernier affluent important et
Durance est récent : au Quaternaire moyen une "capture » vers le Rhône, à travers le goulet d'Orgon, a en effet détourné le cours de la rivière qui s'écoulait auparavant vers la mer par le Col de Lamanon en épanchant ses alluvions et constituant ainsi le vaste glacis caillouteux de la Crau.

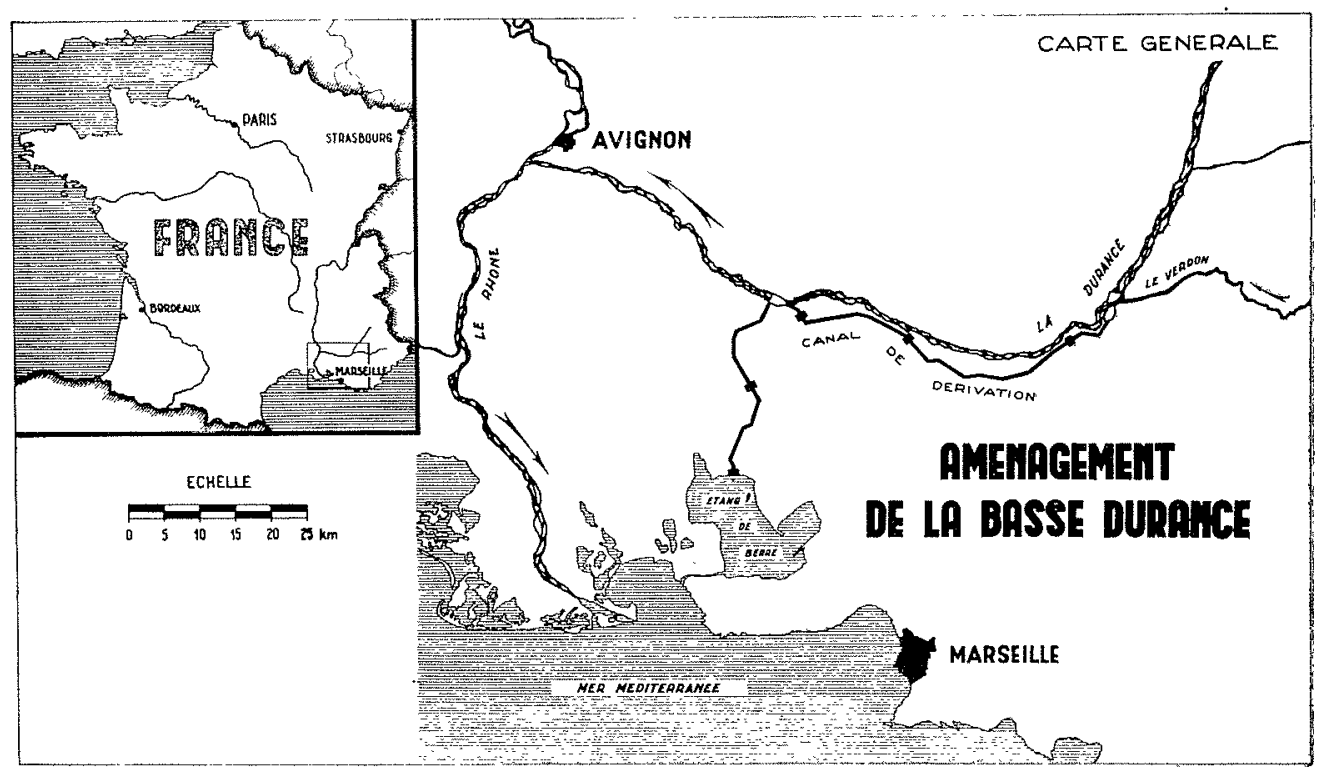

FIG. 1

atteint un module de $200 \mathrm{~m}^{3} / \mathrm{s}$; c'est à partir de là que se développe l'important réseau d'irrigations qui confère à l'agriculture bas-durancienne sa renommée et sa richesse; enfin ce point correspond à l'infléchissement du cours de la rivière qui, quittant le domaine des plissements géologiques alpins (nord-sud), pénètre dans le domaine des plissements pyrénéens ou provençaux (estouest).

I a valléc de la Basse-Durance se développe entre les reliefs du Luberon (qui dépasse $1.000 \mathrm{~m}$ ) situés en rive droite, et la chaîne des Costes prolongée par celle des Alpilles en rive gauche. Schématiquement, la structure géologique de toute cette région se présente sous forme d'une large gouttière synclinale, correspondant à la vallée et qui est limitée au nord et au sud par deux importants bourrelets anticlinaux correspondant aux reliefs et chaînes mentionnés et constitués par des terrains crétacés et tertiaires. Le fond de la gouttière, tapissé par des couches imperméables d'argiles ou de grès très fins, argileux, miocènes ou pliocènes, est rempli par des dépôts alluviaux dont l'épaisseur varie de 5 à $60 \mathrm{~m}$ en amont et de 10 à $30 \mathrm{~m}$ en aval.

Dans sa partie aval, le cours de la Basse-
La plaine alluviale est généralement large, plane, régulière et d'une pente comparable à celle de Ja rivière; ces conditions se sont prêtées à la réalisation d'un réseau d'irrigations alimenté par la rivière elle-même : 14 canaux agricoles, dont le premier remonte au xII" siècle, permettent l'ir" rigation de 75.000 ha. Ces canaux, base de la richesse agricole de la région, possèdent des prises en rivière qui sont soumises aux caprices du tracé du lit mineur de cette derniere, notamment lorsqu'elle connaît son étiage d'été. Les débits ainsi dérivés peuvent atteindre $114 \mathrm{~m}^{3} / \mathrm{s}$, d'après des jaugeages effectués en 1952. Les irrigations constituent donc un apport d'eau considérable sur le sol alluvial de la vallée : dans cet ordre d'idée, il est intéressant de mentionner que près de $40 \mathrm{~m} 3 \mathrm{~s}$ franchissent le col de Lamanon, par les canaux de Craponne et des Alpilles méridionales, pour être répandus sur le sol de la Crau.

La création du réservoir de Serre-Ponçon améliorera indiscutablement la situation souvent grave créée par les sévères étiages d'été de la rivic̀re et les pénuries d'eau agricole qu'ils entraînent (le débit de la Durance peut descendre à $40 \mathrm{~m}^{3} / \mathrm{s}$ en regard des besoins qui, dans le même temps, sont voisins de $90 \mathrm{~m}^{3} / \mathrm{s}$ ); a cet avantage 


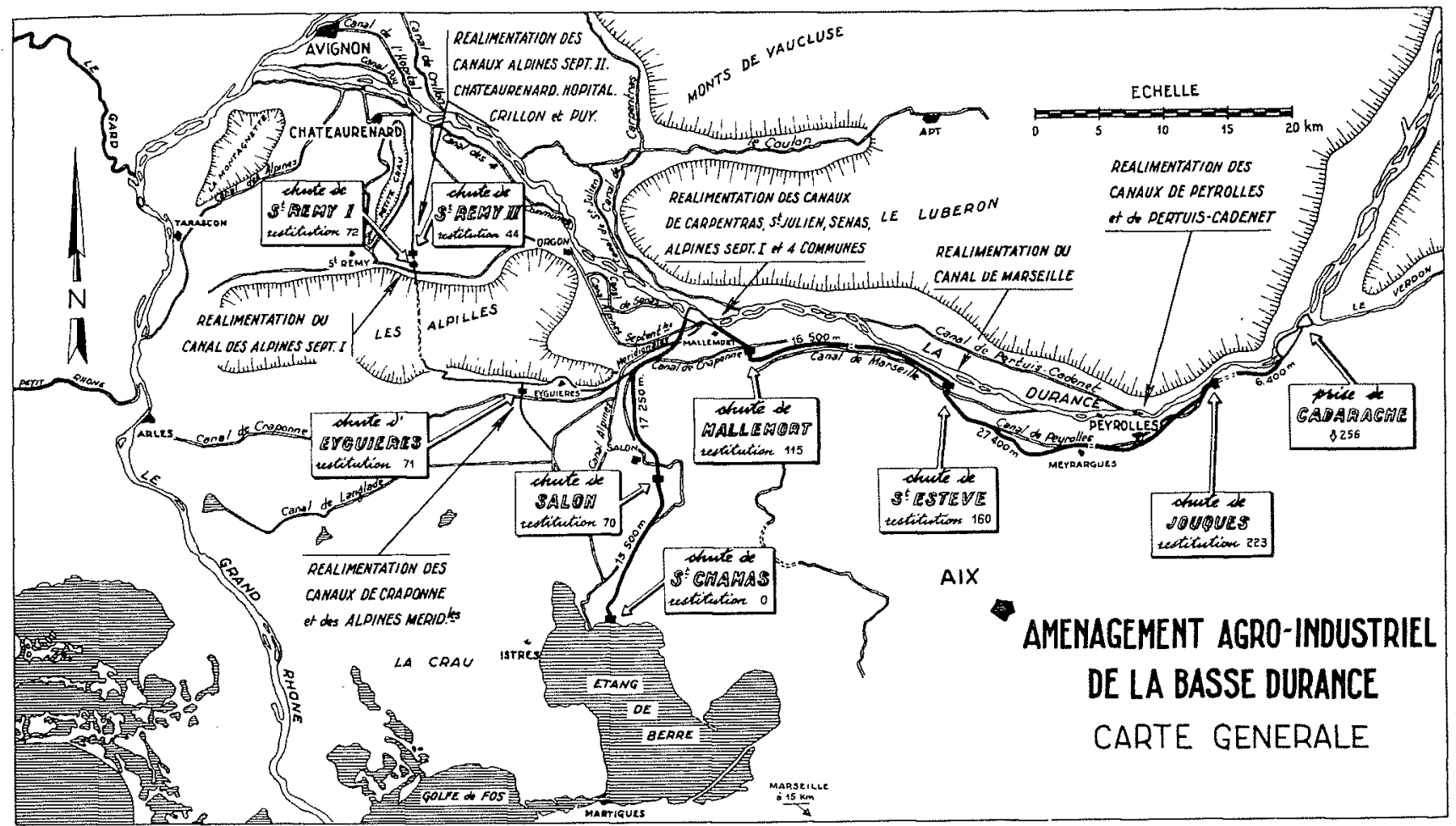

FIG. 2

important viendra s'en ajouter un autre qui, sur un plan différent, n'est pas moindre : celui de l'écrêtement des crues qui, tout en laissant passer encore un milliard de $\mathrm{m}^{3}$ d'eau dans la rivière, atténuera leur violence et entraînera une diminution sensible de leur cortège habituel de dommages.

Toutes ces indications suffisent à suggérer le développement extrêmement poussé de l'agriculture de toute cette région : c'est son importance même qui a entraîné certaines questions concernant les répercussions de l'aménagement de la Basse-Durance sur l'écoulement de la nappe souterraine qu'on rencontre dans les alluvions et, partant sur l'agriculture elle-même. L'aménagement prévoit en effet la dérivation de $250 \mathrm{~m}^{3} / \mathrm{s}$ au niveau du confluent du Verdon (cote 256), un canal industriel de $80 \mathrm{~km}$ débouchant dans l'étang de Berre, et 5 usines (fig. 2) : il profitera, ce faisant, de la forte pente de la rivière $(2,8 / 1.000)$.

Les ouvrages (usines, canal industriel) pourront-ils être situés ou conçus de telle sorte qu'ils ne perturbent pas la nappe alluviale? La réduc- tion sensible des débits qui s'écoulent dans la rivière n'entrainera-t-elle pas une réduction de l'alimentation de la nappe alluviale?

Ces questions ont d'emblée revêtu une très grande importance dans les milieux agricoles régionaux; et les principales oppositions rencontrées par le projet sont nées des idées fausses qui, sans admettre que l'existence même d'irrigations aussi importantes soulignait l'insuffisance ou l'inexistence du rôle de la nappe vis-à-vis des plantes, liaient étroitement la Durance, la nappe et les cultures : n'a-t-on pas entendu parler d'une nappe se présentant comme un fleuve souterrain, d'une région de steppes?... Ce sont ces difficultés qui ont conduit à mettre en lumière avec précision l'importance réelle de la nappe souterraine et les relations qui existent entre la Durance et celle-ci. Les études correspondantes, dont les collectivités locales ont été scrupuleusement informées, ont porté sur deux aspects principaux: d'abord bien connaître toutes les particularités de l'écoulement naturel de la nappe alluviale, ensuite étudier les remèdes techniques à meltre en œuvre pour pallier éventuellement toutes répercussions possibles.

\section{II. - ETUDE GENERALE DE LA NAPPE ALLUVIALE}

Depuis 1946, des observations très complètes, qui sont poursuivies actuellement, portent sur les niveaux statiques de la nappe; elles sont effectuées sur 1.650 points d'eau suivant des fré- 
quences variables : elles peuvent être continues (130 enregistreurs), journalières, hebdomadaires ou mensuelles. Chaque point observé fait l'objet d'une fiche particulière.

Préciser que plus d'un million de lectures de niveau de nappe, de débits en rivière, dans les canaux d'irrigation ou les fossés de drainage, de hauteurs de précipitations... ont été réalisées, a le mérite de fixer l'ampleur de l'opération, mais comporte le risque de masquer l'attention soutenue qu'il a fallu attacher à la qualité de toutes ces mesures pour qu'elles soient exploitables.

Tous ces renseignements, accumulés depuis dix ans, constituent un éfat de référence de la situation ou du régime de la nappe avant tous travaux, état encore appelé « état initial». Ils permettent de poursuivre des recherches en utilisant les méthodes statistiques, sur les relations qui peuvent exister entre les variations de niveau de la nappe et les facteurs d'influence de celle-ci : pluies, irrigations ou crues. Ces relations, si elles pouvaient être définies, permettraient de préciser la part d'influence éventuellement imputable aux travaux par rapport à celle qui serait le fait de la nature elle-même (années sèches ou réduction des irrigations par évolution des cultures, par exemple).

\section{$*$}

Les observations ainsi réunies permettent d'établir tous les documents susceptibles de faire ressortir l'écoulement (fig. 3 et 4), la profondeur, les battements annuels de la nappe (cartes d'isoamplitudes faisant ressortir les zones où les niveaux, très peu variables, sont liés à l'existence d'une condition aval d'écoulement stable), les zones où la nappe est en charge (fig. 5) ou en écoulement à surface libre sous la couche de limons superficiels qui recouvre parfois les alluvions.

De nombreux forages ont par ailleurs fourni les renseignements concernant les paramètres hydrodynamiques qui conditionnent l'écoulement et interviennent dans les calculs : H l'épaisseur des alluvions (précisée également par méthodes géophysiques), K la perméabilité et $m$ la porosité.

La perméabilité a fait l'objet de plus de 1.000 mesures ponctuelles, effectuées le plus souvent par «surcharge, débit-constant»: les coefficients rencontrés varient entre $1.10^{-3}$ et $1.10^{-2} \mathrm{~m} / \mathrm{s}$. Ces mesures ont entraîné diverses recherches : l'une d'elles a ainsi permis de constater que la fonction $h(\mathrm{Q})$ était linéaire dans un certain intervalle et s'infléchissait ensuite soit dans un sens correspondant à un effet de paroi (fuite le long du tube ou cheminement privilégié), soit dans un sens correspondant à un accroissement des pertes de charge. Ce dernier cas étudié a montré que cet infléchissement, observé au-delà de certaines surcharges variables suivant les terrains, correspondait à un nombre de Reynolds systématiquement voisin de 4 , nombre au-delà duquel

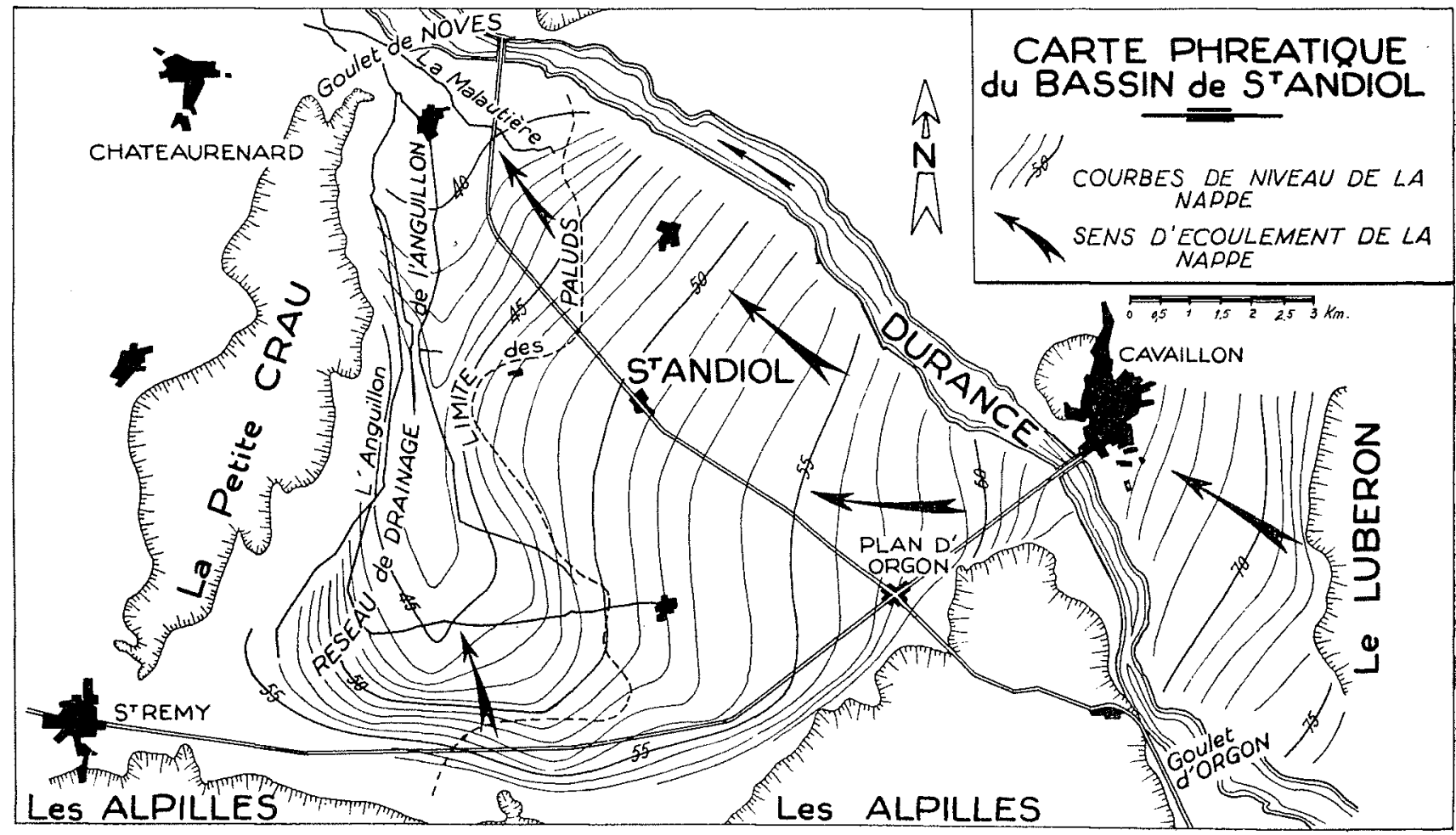

FIG. 3 


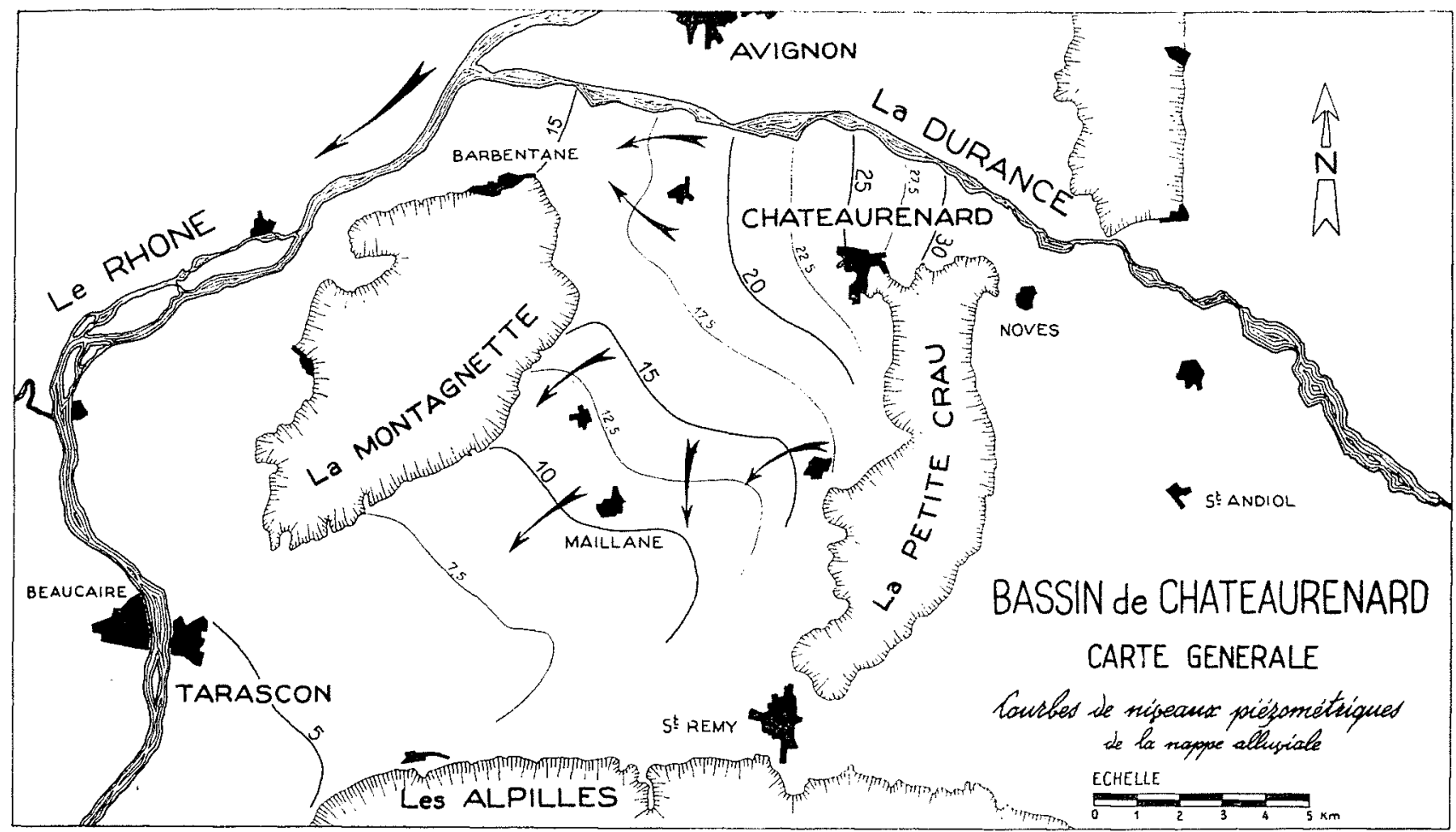

FIG. 4

la loi de Darcy n'est plus rigoureusement applicable.

La porosité a fait l'objet de mesures et d'estimations : la "porosité utile», différente de la «porosité totale » qui est de $24 \%$, s'avère varier entre 6 et $12 \%$ suivant que le terrain est plus ou moins humide au moment de l'injection.

$$
*
$$

Il n'est pas possible de donner ici le détail de Loutes les études de nature variée qui ont été réalisées ni de l'expérience conférée par leur réalisation dans un domaine aussi peu connu et aussi complexe que celui des écoulements souterrains. Aussi nous bornerons-nous à relater les traits principaux de l'écoulement de la nappe avant de poursuivre l'exposé concernant les conséquences de l'aménagement et les palliatifs prévus si besoin est.

De Pont-Mirabeau au Rhône, nous avons vu que la vallée de la Durance est constituée par un remplissage de dépôts alluviaux reposant sur un berceau géologique étanche dont les couches apparaissent parfois sur les reliefs limitrophes; la rivière coule sur ces dépôts alluviaux. La vallée par ailleurs est géographiquement découpée en une suite de bassins séparés par des goulets topographiques qui sont constitués par des resserrements des reliefs voisins et dont les superficies vont en croissant d'amont en aval.
Les observations effectuées sur les niveaux des nappes souterraines montrent que, sur la totalité des versants, ces niveaux se trouvent à une cote supérieure à celle de la rivière : au col de Lamanon notamment, où des écoulements auraient pu se produire du bassin de la Durance vers la Crau, cette disposition s'est trouvée vérifiée et confirmée par des recherches diverses.

Complétant la notion de berceau étanche, on a ainsi la certitude que la nappe alluviale ne s'écoule pas hors du bassin de la rivière.

D'autres observations ont montré que l'écoulement de la nappe alluviale s'effectue suivant des traits qu'on retrouve régulièrement dans chaque bassin (fig. 3):

- Vers le milieu de chacun d'eux, la nappe s'écoule parallèlement à la rivière;

- Vers l'aval, à l'approche du goulet topographique suivant qui entraine un resserrement de la section. d'écoulement, la pente de la nappe diminue et son niveau se rapproche de la surface du sol $(0,30$ à $1 \mathrm{~m})$ entraînant ainsi l'existence des zones de paluds et la nécessité de drainer;

-- A hauteur du goulet, la pente de la nappe s'accroît sensiblement;

- A l'aval du goulet, donc en amont du bassin suivant, le niveau de la nappe est éloigné de la surface du sol (5 à $15 \mathrm{~m})$ et inférieur à celui de la rivière. 
Les relations entre la Durance et la nappe sont donc les suivantes :

- Au milieu et à l'aval des bassins, et à hauteur des goulets, la rivière joue le rôle classique de drain que jouent les rivières à l'égard des nappes latérales : le niveau de la rivière ou celui des réseaux de drainage existants constitue la condition aval d'écoulement de la nappe; celui-ci ne sera pas modifié par la dérivation projetée.

- A l'amont des bassins et sur une zone délimitée, la rivière est en charge par rapport à la nappe : une alimentation à partir de la rivière est dès lors possible. On a observé qu'elle ne peut être sensible qu'en pèriode de crues (accroissement de la surface du champ d'inondation, décolmatages locaux). En dehors de ces périodes, le colmatage naturel du lit de la rivière la réduit considérablement : une telle possibilité d'apport est donc épisodique.

La définition des relations observées dans ces situations de drainage ou d'apport a présenté certaines difficultés lorsqu'il a fallu passer d'une notion qualitative à une notion quantitative.

Une première donnée vient des résultats de bilans effectués pour de faibles débits de la rivière : ceux-ci ont fait ressortir à plusieurs reprises que, entre le confluent du Verdon (station de Pont-Mirabeau) et le confluent du Rhône (Pont de Rognonas), le débit de la Durance, compte tenu des apports latéraux ou des prélèvements agricoles, s'accroît de 10 à $14 \mathrm{~m}^{3} / \mathrm{s}$ : ce débit correspond à l'apport global de la nappe souterraine sur les $90 \mathrm{~km}$ considérés du cours de la rivière.

D'autres évaluations ont porté sur les débits qui peuvent venir de la Durance lorsque celleci a un débit supérieur à $250 \mathrm{~m}^{3} / \mathrm{s}$. Ainsi au droit de la partie amont du bassin de Plan d'Orgon-Saint-Andiol, les relèvements de niveaux de la nappe obtenus en 1952 au cours d'une crue, correspondent à un débit de l'ordre de 400 à $500 \mathrm{l} / \mathrm{s}$ pour une porosité de $8 \%$. Sur l'ensemble de la vallée, un débit épisodique de l'ordre de 1 à $2 \mathrm{~m}^{3} / \mathrm{s}$ a ainsi pu être évalué.

\section{III. - INFLUENCE DES OUVRAGES SUR LA NAPPE ALLUVIALE}

La prise du canal industriel est prévue à quelques kilomètres en amont du Pont-Mirabeau à Cadarache (alt. 256). A partir de ce point, le canal industriel suivra la rive gauche de la vallée de la Durance jusqu'au col de Lamanon (aIt. 112) au-delà duquel il prendra une orientation nord-sud pour gagner l'étang de Berre au voisinage de Saint-Chamas (fig. 2).

Le premier tronçon comportera trois biefs et usines : Jouques (restitution : 225), Saint-EstèveJanson (restitution : 160) et Mallemort (restitution : 115) qui seront situés à proximité du lit de la Durance; de la sorte, les chutes amont pourront être mises en service pendant la réalisation des chutes aval, en restituant provisoirement les eaux turbinées en Durance.

Le second tronçon comportera deux biefs et usines : Salon (restitution : 70) et Saint-Chamas (restitution : 0 ).

La pente de la vallée $(2,8 / 1000)$ est telle que le canal est amené à gagner très rapidement le versant voisin à l'aval immédiat de chaque usine pour pouvoir prendre de la hauteur. Ce faisant, il quitte le domaine alluvial et passe au-dessus d'éventuelles nappes d'eau souterraines sans les affecter.

A l'aval immédiat des usines les cotes de restitution ont été choisies au voisinage des cotes de la nappe de telle sorte que le canal n'entraîne pas de perturbation dans l'écoulement des eaux souterraines. Dans ces zones, le revètement du canal sera perméable afin de permettre des échanges avec la nappe et d'éviter des sous-pressions. Partout ailleurs il sera étanche.

En période de travaux, un drainage de la nappe existera toutefois sur une distance variant entre 800 et $1800 \mathrm{~m}$; ses conséquences pourront être nulles comme par exemple à SaintEstève-Janson du fait de la quasi-absence de nappe.

A Jouques, un canal d'essai silué sur l'emplacement exact et au niveau du futur canal de fuite, a été réalisé dans une zone où la vallée est extrèmement étroite, dans le but d'étudier les effets du drainage sur l'écoulement de la nappe et sur l'alimentation de la nappe du bassin de Peyrolles-Pertuis qui se développe plus en aval.

Les rabattements provoqués ont été en décroissant d'amont $(4 \mathrm{~m})$ en aval $(0 \mathrm{~m})$. La section de la fouille mouillée par la nappe correspond aux $2 / 3$ de celle du canal définitif. Le débit drainé de cette manière est très voisin de celui qui sera obtenu en période de travaux: il a 
atteint $300 \mathrm{l} / \mathrm{s}$ pour une longueur de canal de $1700 \mathrm{~m}$.

Le colmatage naturel du lit de la rivière a été souligné dans une zone où la différence de niveau entre le canal et la Durance distants l'un de l'autre de $40 \mathrm{~m}$, était de $3 \mathrm{~m}$; un profil de sondages a montré en effet que la perte de charge atteignait plus de $2,50 \mathrm{~m}$ à quelques mètres de la Durance $(3 \mathrm{~m})$ pour n'être que d'une vingtaine de centimètres sur le reste de la distance $(37 \mathrm{~m})$.

Les rabattements provoqués se sont très rapi- dement stabilisés : ils se sont limités à une zone située au droit de la fouille et n'apparaissent pratiquement plus à l'aval de celle-ci. On a ainsi pu démontrer que les travaux n'entraîneront aucune perturbation dans l'écoulement de la nappe à l'aval de la fouille et en particulier sur la nappe du bassin de Peyrolles-Pertuis.

En somme, les ouvrages - usines et canaux - ont pu être situés ou conçus de telle sorte qu'ils n'affectent pas l'écoulement nalurel de la nappe.

\section{IV. - DISPOSITIFS D'ALIMENTATION ARTIFICIELLE}

Nous avons vu que l'accroissement du débit s'écoulant de la rivière vers la nappe en période de crues ( 1 à 2 mois par an), était de 1 à $2 \mathrm{~m}^{3} / \mathrm{s}$ pour l'ensemble de la vallée : il a paru possible d'apporter semblables débits à la nappe de manière artificielle pour pallier une éventuelle influence de la dérivation sur ces apports. Il fallait pour cela :

- montrer qu'il était possible d'injecter des débits de l'ordre de ceux qui viennent d'ètre indiqués;

- prouver de façon démonstrative l'efficacité de telles injections;

- donc provoquer une évolution des niveaux plus rapide qu'une évolution naturelle et réaliser pour cela des relèvements sensibles dans un temps court.

La nécessité de conférer un caractère indiscutablement démonstratif à de telles réalisations, a entraîné divers essais et des recherches sur les écoulements souterrains.

Des essais de principe ont ainsi été réalisés en janvier 1953 à Plan-d'Orgon dans le bassin de Saint-Andiol qui est le plus vaste bassin de la vallée, et dans la région d'Arignon à la Seignone.

Leurs résultats ont été largement concluants puisqu'ils ont déterminé l'accord des collectivités locales à l'égard du projet, d'autres essais devant être cependant réalisés pour montrer que, si les dispositifs d'injection devaient être adaplés aux conditions propres à chaque bassin, les effets de l'alimentation artificielle se feraient sentir partout de façon tout aussi efficace.

A loccasion de ces essais, des recherches théoriques ont été entreprises sur les lois qui régissent les écoulements non permanents en milieux poreux: elles ont été traitées par
R. Merer dans une étude de caractère général sur les écoulements en milieux poreux, parue dans la Houille Blanche (1 et 5, 1955 et I, 1956).

Sur le plan pratique, des méthodes de travail ont été élaborées pour mettre en place les dispositifs d'alimentation artificielle de la nappe alluviale suivant les conditions aux limites ou les caractéristiques hydrodynamiques de chaque bassin.

C'est à la lumière de ces recherches et en utilisant ces méthodes que des essais ont été réalisés en 1956 dans les bassins de Chateaurenard et de Peyrolles.

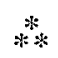

Les essais de Plan d'Orgon ont duré 1 mois au cours duquel un débit moyen de $1 \mathrm{~m}^{3} / \mathrm{s}$ a été injecté : les relèvements obtenus ont ramené les niveaux de la nappe de leur minimum moyen annuel à leur maximum moyen annuel sur la quasi-totalité du bassin intéressé.

Ceux de la Seignone, réalisés à $800 \mathrm{~m}$ en amont de la station de pompage qui alimente Avignon en eau potable, ont démontré qu'ils n'influençaient pas la qualité des eaux pompées : de nombreuses analyses bactériologiques ont souligné qu'un très faible cheminement souterrain $(50$ à $80 \mathrm{~m}$ ) suffisait pour assurer parfaitement l'épuration des eaux injectées.

Les essais de Chateaurenard et de Peyrolles font l'objet de la description qui suit.

\section{1. - L'essai de Chateaunfand}

Le bassin alluvial de Châteaurenard est situé en rive gauche de la Durance avant le confluent de cette rivière avec le Rhône. Il se présente comme une plaine large d'environ $5 \mathrm{~km}$ qui, entre les doux reliefs de la Montagnette, de la Petite Crau et des Alpilles, s'étend depuis la Durance au nord jusqu'à la plaine du Rhône au sud-ouest. Un réseau d'irrigation très développé 
couvre sa partie nord alors que le drainage est systématiquement pratiqué vers l'ouest et le sud (fig. 4).

Ce bassin, ouvert topographiquement vers le Rhône, se présente différemment de celui de Saint-Andiol où avaient été réalisés les premiers essais (fig. 3) : il a donc paru nécessaire de démontrer que cette différence, simplement apparente ainsi que les études ont permis de s'en rendre compte, ne mettait pas en défaut la technique de l'alimentation artificielle.

L'écoulement de la nappe alluviale s'effectue de la facon suivante :

- La nappe s'écoule dans des alluvions dont l'épaisseur peut atteindre $30 \mathrm{~m}$ et qui sont souvent recouvertes par une couche de limons superficiels dont l'épaisseur est variable; celle-ci va en s'accroissant notablement vers l'aval (vers Maillane on en trouve une épaisseur de $14 \mathrm{~m}$ ) alors que dans cette direction la perméabilité des alluvions est sensiblement plus faible (8 à 10 fois environ).

-- Dans la partie amont du bassin, l'écoulement de la nappe est à surface libre jusqu'à une zone où le toit de la nappe rencontre la base de la couche de limons superficiels.

- A l'aval de cette zone, l'écoulement s'effectue en charge sous les limons dans une direc- tion où, comme on vient de le dire, la section d'écoulement et la perméabilité des alluvions diminuent sensiblement.

- Cette limite de mise en charge de la nappe sous les limons est marquée d'une façon continue par des sources et par l'apparition des réseaux de drainage qui évacuent les eaux de débordement de la nappe et ont constitué les premiers travaux d'assainissement dont cette région a été l'objet au $x^{\circ}$ siècle sous la conduite des moines de Montmajour.

Elle correspond donc à une limite au-delà de laquelle l'existence des drains rend impossible tout relèvement du niveau de la nappe : on peut en effet observer que, dans le courant d'une année, les niveaux y varient de 0,20 à $0,30 \mathrm{~m}$ environ du fait de l'exploitation des drains, alors qu'ils varient naturellement de plus de $1 \mathrm{~m}$ dans toute la partie amont du bassin.

\section{i) Caractéristiques DE L'Essai :}

L'essai d'alimentation artificielle a porté sur la zone ainsi délimitée dans laquelle la profondeur de la nappe varie d'amont en aval de $4 \mathrm{~m}$ à $2 \mathrm{~m}$ (fig. 5). Le dispositif d'injection a été mis en place à la lumière des études et selon les méthodes précédemment évoquées; les carac-

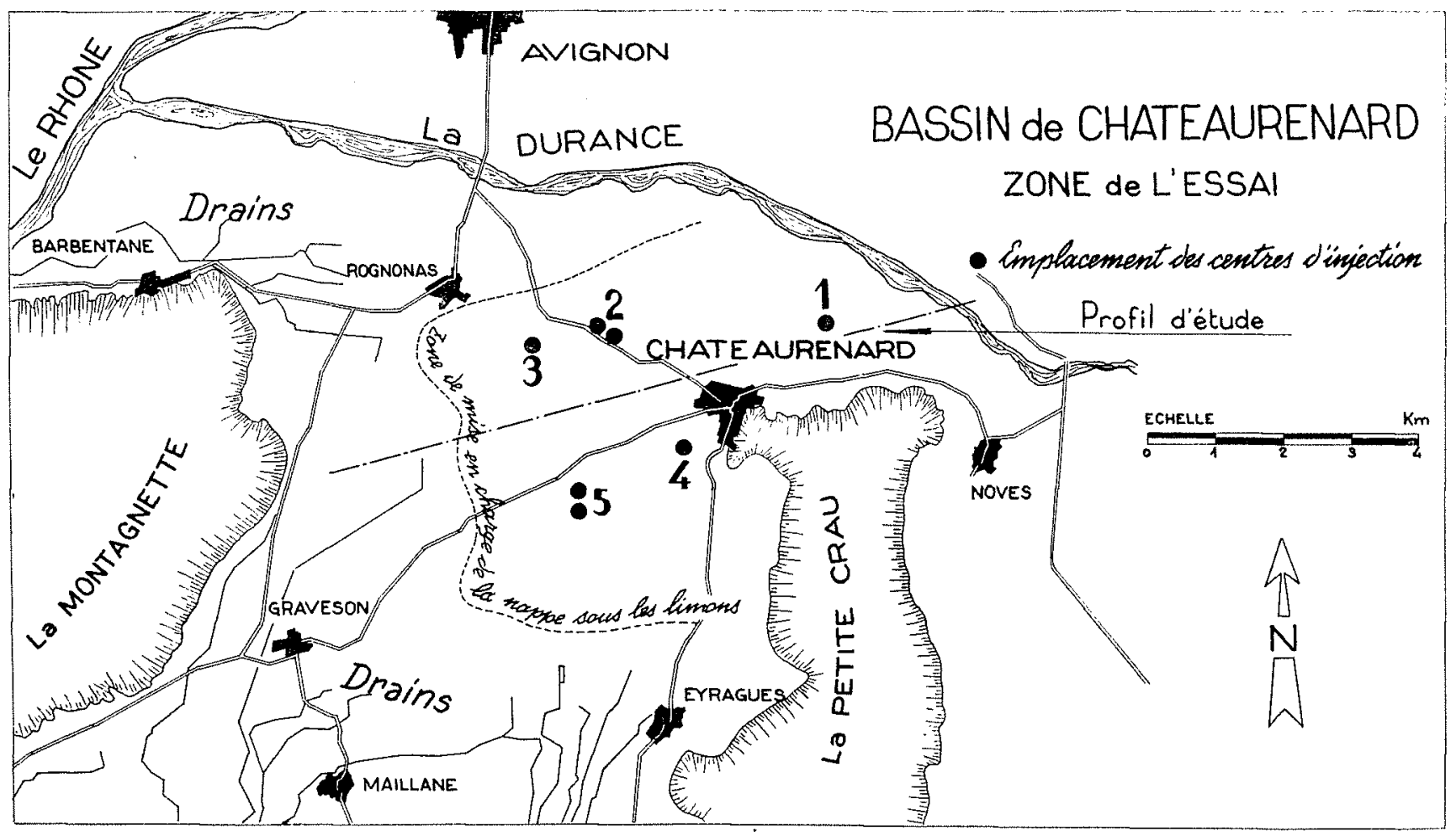

FiG. 5 


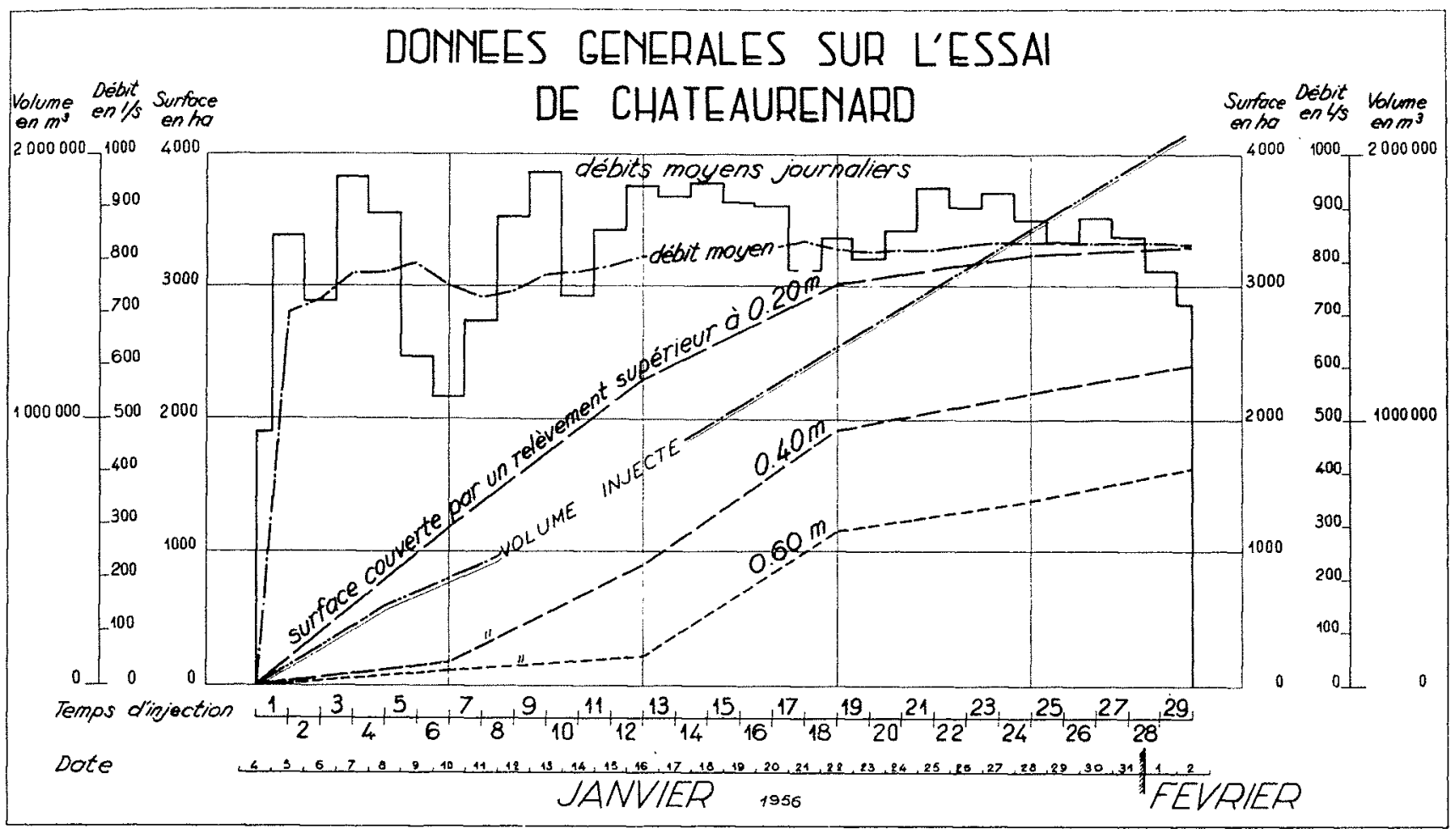

Fic. 6

téristiques hydrodynamiques retenues pour les calculs sont les suivantes :

Epaisseur de la nappe...... H $=20 \mathrm{~m}$ Perméabilité des alluvions.... $K=8.10^{-3} \mathrm{~m} / \mathrm{s}$ Porosité des alluvions.......m $m=6$ a $12 \%$

Après ajustements successifs, les recherches ont abouti à un dispositif constitué par 5 centres équipés pour absorber $1100 \mathrm{l} / \mathrm{s}$. Ces centres comportent des ouvrages de filtration (l'eau d'amenée étant l'eau de Durance distribuée par les canaux d'irrigation) de deux types (100 et $200 \mathrm{l} / \mathrm{s}$ ) et des ouvrages d'injection (25 sondages de $560 \mathrm{~mm}$ foncés jusqu'au fond imperméable).

Pour que de tels essais soient probants, il fallait les réaliser dans des conditions très sélectives, c'est-à-dire en une période où les crues et les pluies sont très peu probables et les irrigations interrompues; aussi, comme les essais de principe de 1953, ceux de Châteaurenard et de Peyrolles (1956) ont été réalisés en janvierfévrier. A ce moment par ailleurs, les niveaux de la nappe sont généralement à leurs minima annuels: celte situation permet de provoquer des relèvements sensibles sans entrainer des dommages liés à des niveaux élevés inhabituels.

\section{b) Résultats de L'essai :}

L'essai s'est poursuivi 29 jours. Le débit moyen d'injection a été maintenu au voisinage de $830 \mathrm{l} / \mathrm{s}$ : plus de 2000000 de $\mathrm{m}^{3}$ d'eau ont été ainsi injectés. Ces données apparaissent sur la figure 6 qui présente également l'évolution des surfaces couvertes par des relevements supérieurs à $0,20,0,40$ et $0,60 \mathrm{~m}$.

Au cours de l'essai, une crue de la Durance a été observée. Aussi, pour connaitre l'efret réellement imputable à l'essai, il a fallu reconstituer par corrélalions graphiques, à partir des niveaux observés sur des puits comparables mais non soumis à l'influence de l'alimentation artificiel'e, ce qu'aurait élé l'évolution naturelle des niveaux. Ce sont les différences de niveaux lues sur ces graphiques qui ont permis de tracer la coupe en long du bassin (fig. 7) et les cartes des relèvements conséculifs à l'injection. La figure 8 compare les résultats calculés et en fonction desquels le dispositil a été mis en place, et les relèvements observés à 29 jours.

\section{2. - L'essai de PEymolles}

Entre la chute de Jouques et la chute de SaintEstève-Janson et en rive gauche de la Durance, s'étend le bassin de Peyrolles. Celui-ci présente des caractéristiques générales comparables à celles du bassin de Châteaurenard: elles ont conduit, de la même façon qu'à Châteaurenard, a réaliser l'essai dans la partie amont du bas- 

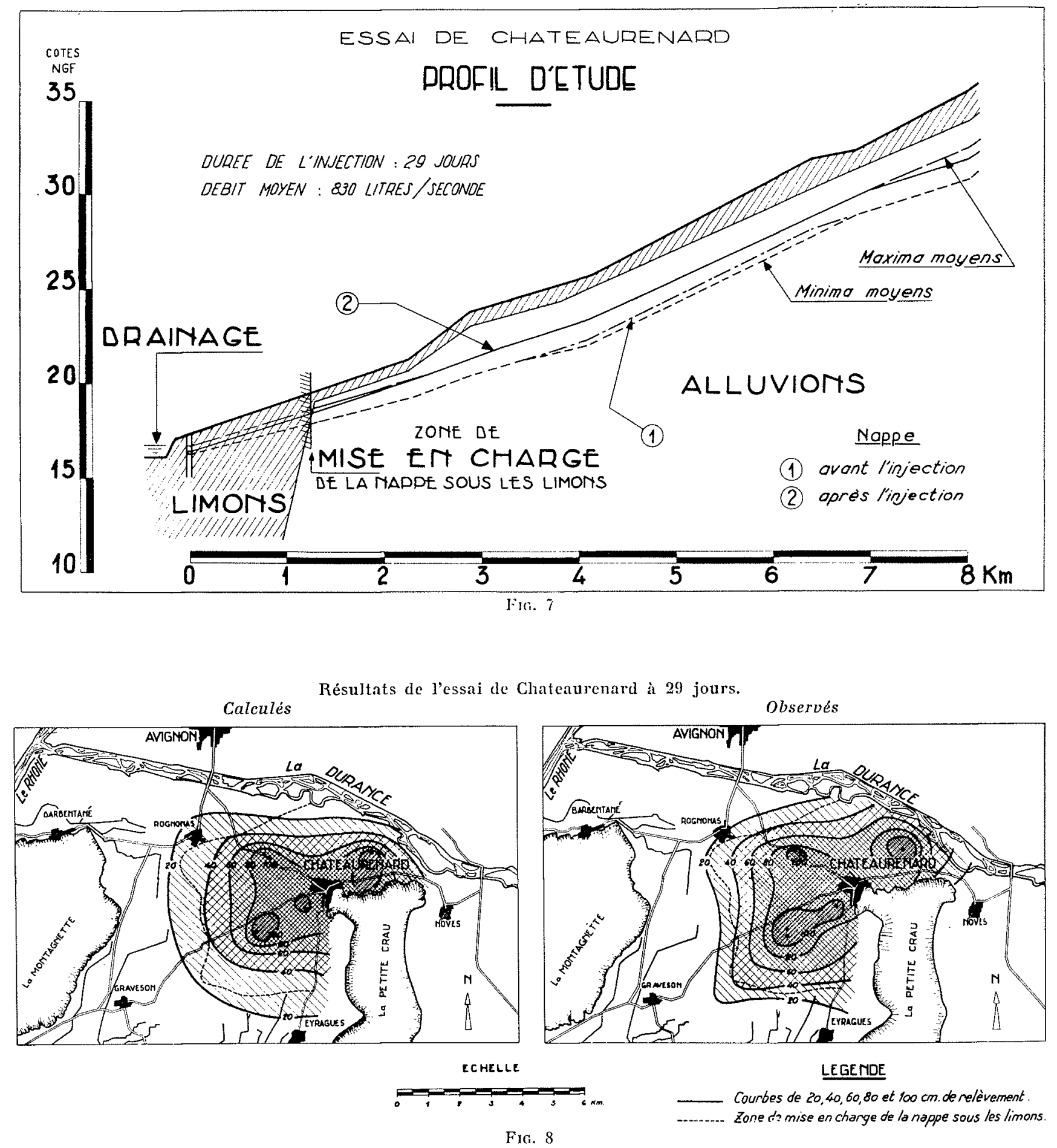

sin (fig. 9). On trouve en effet dans cette région :

- La nappe alluviale qui s'écoule librement au droit de Peyrolles, dans une zone où les irrigations sont très développées.

- Une zone de mise en charge de cette nappe sous des limons superficiels.
- L'existence d'un réseau de drainage qui limite tous relèvements à l'aval de cetle zone.

Complétant cette limite aval de l'essai, on trouve, en amont de Peyrolles et à $500 \mathrm{~m}$ à l'est du ruisseau de Jouques, un haut fond molassique sur lequel la nappe est pratiquement inexistante et qui constitue une limite amont. 


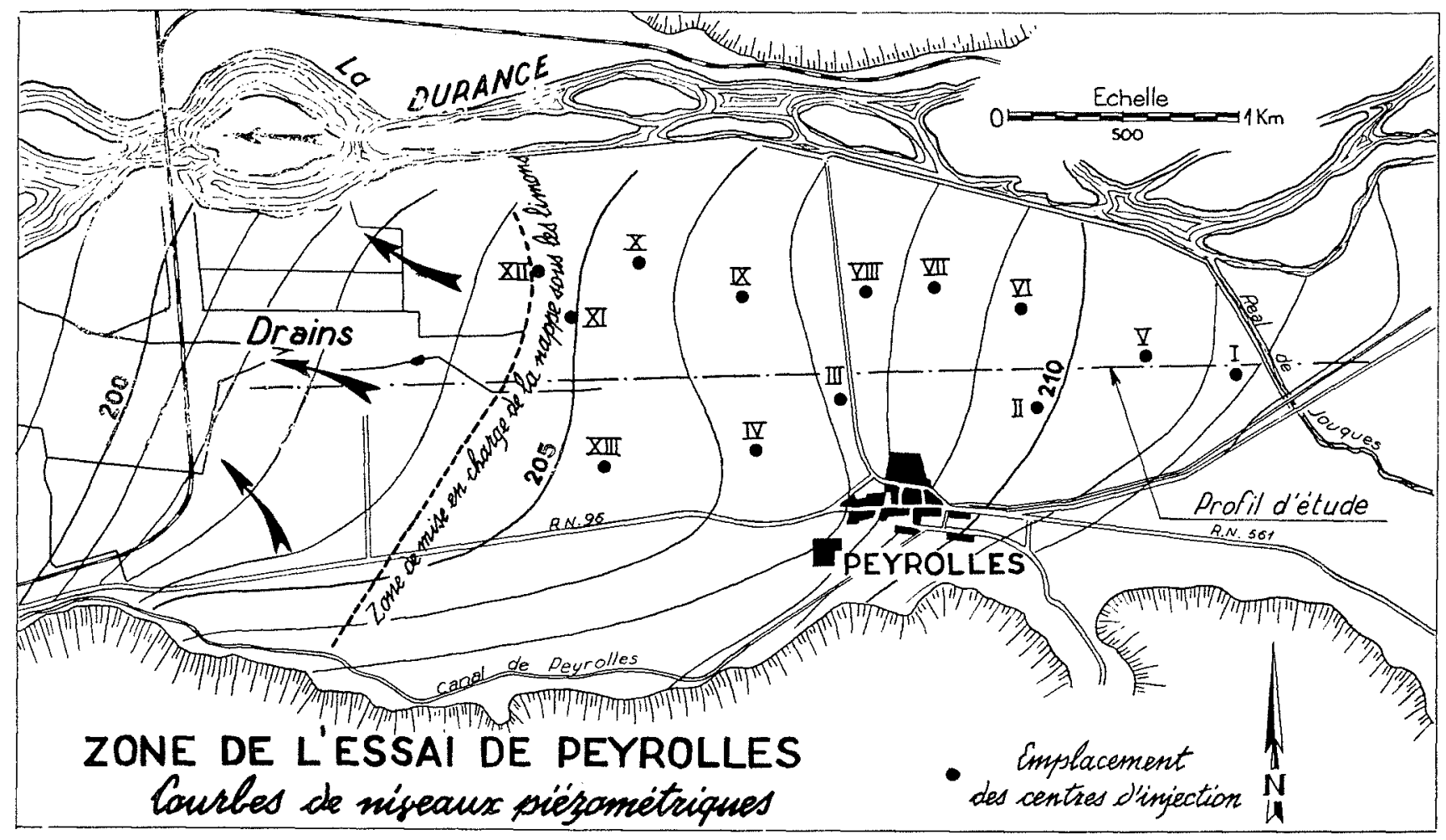

Fic. 9

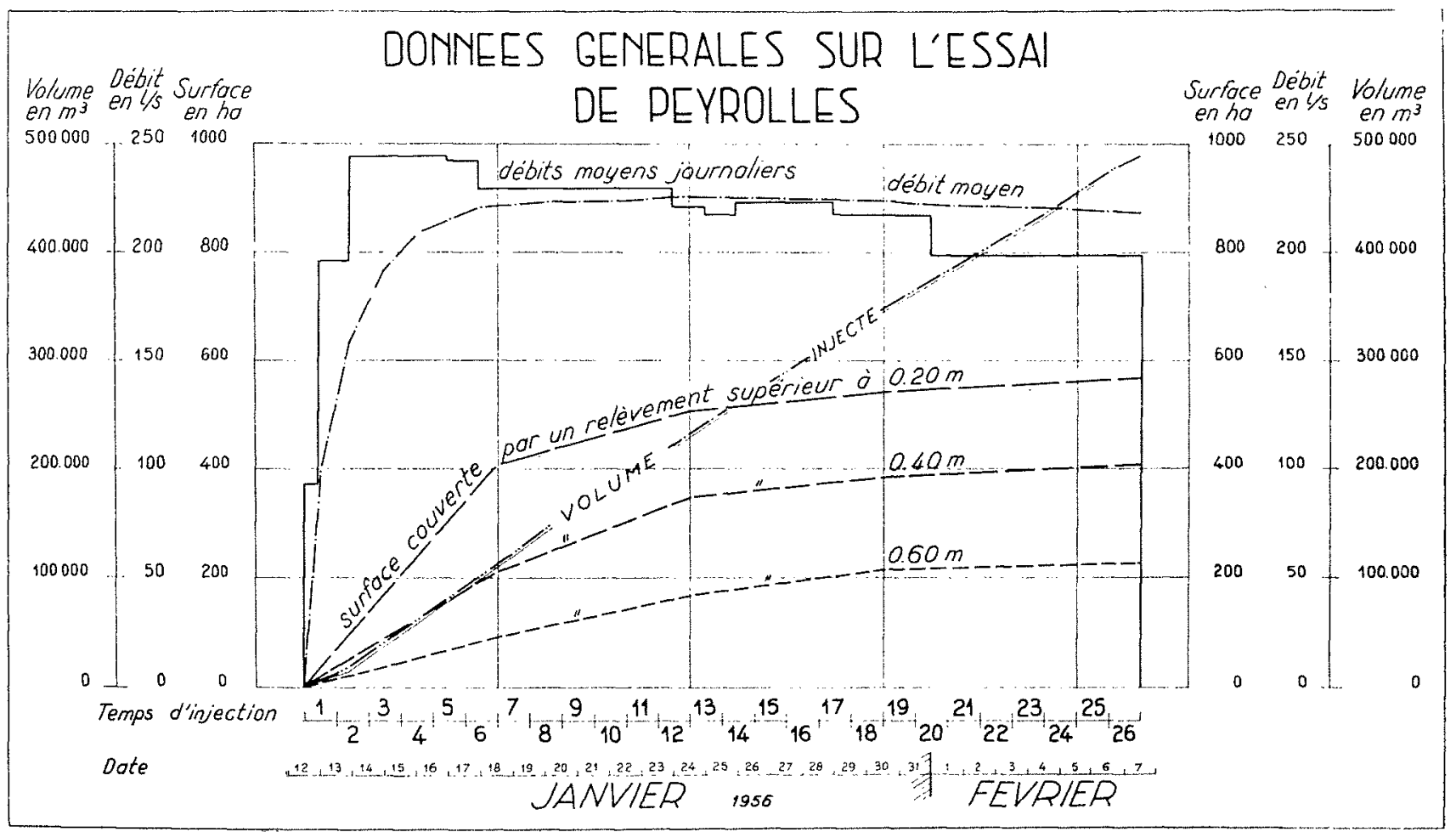

Fig. 10

a) Caractéristiques de L'essai :

Il a porté sur la zone ainsi délimitée dans laquelle la profondeur de la nappe sous le sol varie d'amont en aval de $3 \mathrm{~m}$ à $0,80 \mathrm{~m}$.
Par rapport à la première étude, des différences sensibles apparaissent dans la valeur de certains paramètres : du même coup le dispositif d'injection est assez différent. 
Les caractéristiques retenues pour les calculs sont :

Epaisseur de la nappe.......H $=8 \mathrm{~m}$

Perméabilité des alluvions.... $\mathrm{K}=5.10^{-3} \mathrm{~m} / \mathrm{s}$ Porosité des alluvions.......m $m=6$ à $12 \%$

Le dispositif finalement adopté comporte 13 sondages $(560 \mathrm{~mm})$ foncés jusqu'au fond sous-alluvial et destinés à injecter $240 \mathrm{l} / \mathrm{s}$ au total. Il a été possible de s'affranchir des ouvrages de filtration du fait de l'existence d'eau limpide provenant du ruisseau de Jouques voisin.

\section{b) Résultats de L'essai :}

Il a duré 26 jours. Le débit moyen d'injection s'est fixé à $220 \mathrm{l} / \mathrm{s}$ : près de $500000 \mathrm{~m}^{*}$ d'eau ont été injectés (fig. 10).

D'une manière générale, ces essais ont souligné les conclusions qui étaient apparues au cours des essais de principe de 1953:

- Un débit même supérieur à $1 \mathrm{~m}^{3} / \mathrm{s}$ peut très facilement être injecté dans la nappe alluviale;

- Les ouvrages d'injection sont extrêmement modestes;

- Les relèvements de niveau obtenus en un mois dans chacun des cas et avec les débits respectifs mentionnés, sont partout ceux que l'action conjuguée des facteurs naturels produit en un cycle annuel;

- Ces relèvements, au bout du délai d'un mois indiqué, continueraient à se poursuivre linéairement sans donner de signe de fléchissement: les débits à mettre en œuvre pour maintenir la nappe à un niveau déterminé seraient donc très sensiblement plus faibles que ceux qui ont été utilisés.

\section{V. - CONCLUSIONS}

$\mathrm{Si}$ la technique spéciale de l'alimentation artificielle d'une nappe aquifère a fait l'objet d'applications antérieures aux essais décrits, ces derniers constituent cependant une étape importante du fait des exigences très sévères qu'ils ont dû satisfaire. Tant sur le plan pratique que sur le plan psychologique, sans préjuger de la nécessité d'y avoir recours dans l'avenir, ils ont tranquillisé tous les intéressés car ils ont fait apparaître que, malgré la complexité des questions d'écoulements souterrains qui, par cela même sont mal connues, on disposait d'un palliatif certain en cas d'une éventuelle modification de l'écoulement de la nappe alluviale imputable à la dérivation de la Durance.
Sur le plan général de l'hydrologie souterraine, ils ont montré que des études théoriques, généralement ardues dans ce domaine, peuvent parvenir à une approximation très satisfaisante.

Qu'il s'agisse des recherches d'ordre général sur les écoulements souterrains, des recherches statistiques qui visent la définition d'un «état initial » ou de celles qui concernent les essais d'alimentation artificielle, les questions qui viennent d'être très brièvement évoquées constituent à vrai dire des ensembles distincts : ceuxci mériteraient un développement particulier si on voulait faire ressortir les enseignements qui découlent des études de nappe liées à l'aménagement de la Basse-Durance.

\section{DISCUSSION}

Président : M. BERcieron

Répondant à une question de M. Deymí, M. MullerFeuga indique que la zone intéressée par l'essai d'alimentation artificielle de la nappe (essai de Plan d'Orgon, 1953) a un rayon global d'environ $4 \mathrm{~km}$ : il est évident toutefois que cette distance, valable pour un débit d'injection de $1 \mathrm{~m}^{3} / \mathrm{s}$, varie aulour de la valeur indiquée, suivent la direction considérée du fait de la pente de la nappe et de l'influence des limites imperméables du bassin alluvial.

Répondant à une question de M. Davidenkofr, M. Muller-Feuga indique que, pour l'étude de la limite de validité de la loi de Darcy pour laquelle on a trouvé un nombre de Reynolds égal $\dot{a} 4$, le « diamètre équi- valent " du matériau alluvial était pris égal à $d_{10}=0,18 \mathrm{~mm}$.

Répondant à M. Gurliot, M. Muller-Feuga indique que le coefficient de "porosité utile » des alluvions de la Durance a été évalué en laboratoire comme étant de l'ordre de $8 \%$ en regard d'une « porosite totale» de 24. \%. En procédant par un analyse des essais d'alimentation artificielle, e'est-à-dire en faisant les hypothèses sur 2 ou 3 paramètres qui conditionnent des essais, les autenrs ont été amenés à trouver que le coefficient de porosité à considérer était de l'ordre de $10 \%$ pour le matériau alluvial.

M. Ie Président remercie MM. Decelle et MullerFEuga de leur communication. 